\title{
Discussion on critical points for a tailored therapy to cure hepatitis $C$ virus infection
}

\author{
Nadia Marascio', Angela Quirino', Giorgio Settimo Barreca', Luisa Galati', Chiara Costa², Vincenzo Pisani², \\ Maria Mazzitelli', Giovanni Matera', Maria Carla Liberto', Alfredo Focà', and Carlo Torti ${ }^{2}$ \\ 'Department of Health Sciences, Unit of Clinical Microbiology and ${ }^{2}$ Department of Medical and Surgical Sciences, Unit of Infectious and \\ Tropical Diseases, Magna Graecia University, Catanzaro, Italy
}

Hepatitis C virus (HCV) infects around 71 million people worldwide and in 2018 it is still a major health problem. Since 2011, anti-HCV therapy with availability of direct-acting antiviral drugs has revolutionized the clinical response and paved the way to eradication strategies. However, despite the high rate of sustained virological response, treatment failure may occur in a limited percentage of patients, possibly due to resistance-associated substitutions (RASs), either emergent or pre-existent even in minority viral populations. Clearly this problem may impair success of eradication strategies. With this background, several questions marks still exist around HCV treatment, including whether pan-genotypic treatments with complete effectiveness in any clinical conditions really exist outside clinical trials, the actual cost-effectiveness of genotyping testing, and utility of RAS detection in viral quasispecies by next generation sequencing approach. In this review, we describe these critical points by discussing recent literature data and our research experience. (Clin Mol Hepatol 2019;25:30-36)

Keywords: Direct-acting antivirals; Resistance-associated substitutions; Genetic variation; Deep sequencing

\section{INTRODUCTION}

Hepatitis C virus (HCV) is a single-stranded, positive-sense RNA virus, infecting approximately 71 million people worldwide.' The World Health Organization estimated the elimination of HCV infection by 2030, but this assumption is limited mainly by global HCV epidemiology and financial availability of drugs in different countries. $^{2}$

HCV displays a high genetic diversity and is classified now into eight genotypes, ${ }^{3}$ at least 67 subtypes, ${ }^{4}$ isolates and quasipecies. ${ }^{5}$ Geographic distributions of HCV types vary according to epidemi- ological factors. Even if data on HCV epidemiology are limited and restricted to hospital or regional levels, Kartashev et al. reported an update of genotype/subtype distribution based on data from 52 centers, routinely diagnosing HCV infection. ${ }^{6}$ Between 2011 and 2015, the most prevalent was HCV genotype 1 (GT1), followed by GT3 and GT4 in West European (including Italy), Russian and Israeli regions. ${ }^{6-8}$

In the last seven years, therapy for HCV improved with the availability of several direct-acting antiviral (DAA) drugs, which allowed to skip the use of PEGylated-interferon (PEG-IFN) and ribavirin (RBV). ${ }^{9}$ Indeed, DAA combinations confer good effective-

\footnotetext{
Abbreviations:

DAA, direct-acting antiviral; HCV, hepatitis C virus; LiPA, line probe assay; NGS, next generation sequencing; PEG-IFN, PEGylated-interferon; RAP, substitutions at RAS position; RAS, resistance-associated substitutions; RBV, ribavirin; SOF, sofosbuvir; SVR, sustained virological response; VEL, velpatasvir; VOX, voxilaprevir; 3D, ombitasvir/paritaprevir/ritonavir plus dasabuvir
}

\author{
Corresponding author : Nadia Marascio \\ Department of Health Sciences, Unit of Clinical Microbiology, Magna \\ Graecia University, Viale Europa, Loc. Germaneto, Catanzaro 88100, Italy \\ Tel: +39-961-369-7749, Fax: +39-961-369-7760 \\ E-mail: nadiamarascio@gmail.com \\ https://orcid.org/0000-0003-0880-8955
}


ness and safety for both treatment-naive and previously treated patients in more than $95 \%$ of patients achieving sustained virological response (SVR). ${ }^{10}$ Despite the high rate of SVR with DAAs, non-response to IFN-free regimens (5\%) may be due to resistanceassociated substitutions (RASs) specific for each genotype/subtype. ${ }^{10,11}$ RAS are amino acid (AA) changes on DAA target regions, either pre-existing or selected by drug pressure and associated with a reduced susceptibility to the administered drugs. ${ }^{12,13}$ When detected in more than $15 \%$ of the burden of the entire viral population, RASs play an important role for therapy outcome, ${ }^{10}$ although the clinically meaningful cut-off is not validated yet. In this review, we focused our attention on the main questions in the field of clinical virology of HCV treatment nowadays (Table 1).

\section{FIRST QUESTION: “ARE CLINICAL TRIALS FULLY INFORMATIVE ON PAN-GENOTYPIC EFFECTIVENESS OF NEW REGIMENS IN THE REAL CLINICAL SETTING?"}

DAAs target nonstructural proteins, including NS3/4A, NS5A and NS5B polymerases. ${ }^{14}$ In 2011, the first-wave, first-generation of protease inhibitors boceprevir and telaprevir was approved in combination with PEG-IFN and RBV to treat chronic HCV GT1 infection. In 2013, a second-wave, first-generation of protease inhibitors, such as simeprevir and sofosbuvir, a nucleotide polymerase inhibitor, was approved or use in combinations, with or without PEG-IFN. Starting from 2014, IFN-free combinations were broadly used across European Union. ${ }^{15}$ In Asian countries, such as Korea, boceprevir was approved in May 2014, while several DAA IFN-free regimens have been approved up to November 2015 for the treatment of chronic hepatitis $C^{13}$ Some IFN-free regimens may provide broad coverage for all six major genotypes after 12 weeks of treatment, ${ }^{16}$ however according to drugs availability on the different countries. ${ }^{17}$ Lastly, in 2017, the US Food and Drug Administration approved a pan-genotypic therapy with a high ge- netic barrier to resistance ${ }^{18}$ that is the number of nucleotide changes requested to develop resistance.

However, efficacy of combination therapy appears to be influenced by HCV genotype/subtypes, carrying RAS at specific AA positions for each genomic region. ${ }^{12}$ Several studies showed different regional prevalence of natural RASs. ${ }^{19-21}$ For instance, AA sequences corresponding to targets of protease inhibitors are different in HCV GT3 compared with HCV GT1b. Indeed, HCV GT3 isolates carry the natural resistance D168Q in NS3 region. Also, the Q80K with high prevalence among HCV GT1a isolates reduced response in infected patients, ${ }^{19}$ as well as the $C 316 \mathrm{~N}$ variant associated with resistance to NS5B inhibitors was observed as natural polymorphism at different frequencies in isolates from Asia (54\%), USA (28\%) and Europe (20\%). ${ }^{20}$ The $\mathrm{Y} 93 \mathrm{H}$ related to resistance to all NS5A inhibitors showed lower frequency in HCV GT1b in patients from China (5.08\%), Europe (15\%) and USA (9.3\%). ${ }^{21}$ This RAS was observed in half patients at baseline before asunaprevir plus daclatasvir treatment failure ${ }^{22}$ and it was most important predictive factor of SVR in Asian countries. ${ }^{23}$ Additionally, genetic variability of HCV types impacts on RAS selection. ${ }^{24}$ Among IFNfree therapy, $3 D \pm R$ regimen (ombitasvir/paritaprevir/ritonavir plus dasabuvir with or without RBV), showed different resistance profiles depending on subtypes. In fact, on one side, D168V in NS3, and M28T+Q30R in NS5A, and S556G in the NS5B region were detected on HCV GT1a strains at the time of failure. ${ }^{25}$ On the other side, an HCV GT1b positive patient, naïve to all HCV treatment, carried the acquired NS5A Y93H and NS5B S556G RASs in two out of three regions at the time of 3D-R failure. ${ }^{26}$

Although new pan-genotypic combinations have been approved, solid, real-world data with these combinations in the diverse clinical scenarios and for all genotypes deserve to be collected. Also, in Table 2 we report some of clinical trials and SVR percentages according to genotypes/subtypes of HCV, showing that even in clinical trials small differences among genotypes exist. Such small differences could easily translate into major differences in absolute terms of patients who will fail when these regi-

Table 1. Major question marks

\begin{tabular}{|c|c|}
\hline Question number & Question text \\
\hline First & $\begin{array}{l}\text { Are clinical trials fully informative on pan-genotypic effectiveness upon all HCV genotypes/subtypes in the real clinical } \\
\text { setting? }\end{array}$ \\
\hline Second & Is resistance testing still needed notwithstanding the high genetic barrier of current anti HCV drugs? \\
\hline Third & $\begin{array}{l}\text { Is next generation sequencing able to detect resistance-associated substitutions in minor viral populations useful for } \\
\text { clinical practice? }\end{array}$ \\
\hline
\end{tabular}

HCV, hepatitis C virus. 
mens are used extensively in clinical practice (Table 2).

Sofosbuvir/velpatasvir/voxilaprevir (SOF/VEL/VOX), a fixed-dose combination of NS5B, NS5A and NS3 protease inhibitors, was approved for treatment of HCV GT1-6 infections. Particularly, this regimen is indicated for NS5A inhibitor-naïve patients infected by HCV GT1a and HCV GT3. However, it is not recommended for patients with moderate-to-severe cirrhosis, because safety and efficacy have not been evaluated in patients with decompensated cirrhosis. ${ }^{27}$ Glecaprevir (protease inhibitor) plus pibrentasvir (NS5A inhibitor) regimen showed a high barrier against resistance in NS3 and NS5A target regions, ${ }^{28}$ and it is also suggested as a valid therapeutic option for patients with comorbidities, such as advanced stage chronic kidney disease. ${ }^{29}$ However, a recent HCV GT6a in vitro model showed how virus "escapes", with emergence of RASs for VEL (L31V in NS5A), and for SOF (S282T in NS5B), facilitating selection of L28S RAS in NS5A under pibrentasvir therapy. ${ }^{30}$ So, HCV GT6a and HCV GT3a may have a lower genetic barrier against emergence of resistance to this drug when compared with HCV GT1a. ${ }^{30}$ Finally, SOF/VEL with or without VOX are associated with high efficacy and improvement in patient-reported outcomes scores according to POLARIS-2 and POLARIS-3 clinical trials. ${ }^{31}$

DAA classes are characterized by different degree of genetic barrier against the emergence of viral resistance to drugs, which is related to viral characteristics, such as target region and genotypes/subtypes. ${ }^{24}$ In fact, as discussed above, some HCV types are more prone to lower susceptibility to IFN-free regimens than others. In 2016, Polilli et al. reported a decrease in cost-effectiveness of DAA treatment due to decreased efficacy because drugs were chosen based on inaccurate genotyping by line probe assay (LiPA) assay. ${ }^{32}$ Cost of DAAs is still a limitation that may reduce cost-effectiveness of DAA treatment compared with PEG-IFN plus RBV. ${ }^{33}$ If genotyping still has an impact on treatment choice and effectiveness of this treatment, then its importance for clinical practice is demonstrated. The seven major genotypes are globally distributed according to risk factors and new migration route in different countries. HCV GT1, HCV GT2 and HCV GT3 show a widespread distribution in almost all parts of the world. HCV GT4 is restricted to few countries, such as Middle East, Africa, Saudi Arabia, Egypt and Ethiopia. HCV GT5 has been reported in South Africa, HCV GT6 in the South-East Asia and HCV GT7 in the Central Africa. ${ }^{34}$ In 2012 we created the South Italian Network for Rational Guidelines and International Epidemiology (SINERGIE) project to improve care delivery through integration of clinical, epidemiological, virological and biostatistics expertise. ${ }^{35}$ In the Calabria Region, HCV GT1b was found to be the most prevalent (49.2\%) followed by HCV GT2a/2c (22.4\%), by HCV GT3 (7.4\%) and HCV GT4 (6.2\%). Therefore, the dynamics of HCV genotypes distribution showed overall a decrease of HCV GT1b and an increase of HCV GT4 from 2011 to 2013, ${ }^{36}$ and, particularly, in a small town surveyed in 1996 and in 2010 it was shown that HCV GT2 became the most prevalent HCV type. ${ }^{37}$ Given the diversity and evolution of genotypes in different contexts, determination of the infecting genotype in a patient cannot be avoided.

\section{SECOND QUESTION: "ARE RESISTANCE AS- SAYS IMPORTANT IN CLINICAL PRACTICE?"}

In this scenario, HCV genotype should be detected by NS5B se-

Table 2. Summary of best and worst response of hepatitis C virus genotypes/subtypes to the so called "pan-genotypic" regimens from selected clinical trials

\begin{tabular}{|c|c|c|c|c|c|c|c|}
\hline \multirow{3}{*}{ Therapy } & \multicolumn{4}{|c|}{ Treatment response } & \multirow{3}{*}{$\begin{array}{c}\text { Treatment } \\
\text { duration } \\
\text { (weeks) }\end{array}$} & \multirow{3}{*}{$\begin{array}{l}\text { Treatment } \\
\text { status }\end{array}$} & \multirow{3}{*}{ Clinical trials } \\
\hline & \multicolumn{2}{|c|}{ Best } & \multicolumn{2}{|c|}{ Worst } & & & \\
\hline & SVR (N/N [\%]) & $\begin{array}{l}\text { Genotype/ } \\
\text { subtype }\end{array}$ & SVR (N/N [\%]) & $\begin{array}{l}\text { Genotype/ } \\
\text { subtype }\end{array}$ & & & \\
\hline SOF/VEL/VOX & $146 / 150(97)$ & 1 & $74 / 78(95)$ & 3 & 12 & Experienced & POLARIS-1 ${ }^{27}$ \\
\hline SOF/VEL/VOX & $54 / 53(98)$ & 1 & $51 / 54(94)$ & 2 & 12 & Experienced & POLARIS- $4^{27}$ \\
\hline SOF/VEL & 33/21 (97) & $1 b$ & $44 / 52(85)$ & 2 & 12 & Experienced & POLARIS- $4^{27}$ \\
\hline SOF/VEL/VOX & 91/92 (99) & 3 & 155/169 (92) & 1 & 8 & Naïve & POLARIS-2 $2^{31}$ \\
\hline SOF/VEL & 170/172 (99) & 1 & $86 / 89$ (97) & 3 & 12 & Naïve & POLARIS- $2^{31}$ \\
\hline$G / P$ & 142/145 (98) & 2 & 43/46 (93) & 4 & 8 & Naïve & SURVERIOR-II' \\
\hline
\end{tabular}

SVR, sustained virological response; SOF, sofosbuvir; VEL, velpatasvir; VOX, voxilaprevir; G/P, glecaprevir/pibrentasvir; Experienced, previous direct-acting antiviral therapy; Naïve, previously not treated or treated only with PEGylated-interferon plus ribavirin. 
Nadia Marascio, et al. Tailored therapy to cure HCV infection

quences analysis, which also allows detection of RASs. In fact, reverse hybridization LiPA is not able to determine the correct subtype. $^{38}$ Stelzl et al. reported a new real-time polymerase chain reaction assay including genotype and subtype specific primers for amplification of parts of $5^{\prime} U T R$, Core, and NS5B regions. This new assay allows correct determination of HCV GT1 to HCV GT4 and is able to discriminate HCV GT1a from HCV GT1b. ${ }^{39}$ Viral genome sequencing is the gold standard for HCV type identification. In the last 5 years, during phylogenetic and molecular characterization of HCV, we compared results from routinely used LiPA assay and NS3/NS5B sequences analysis. Twenty-five isolates detected as HCV GT4a/4d/4d and 54 samples originally typed as HCV GT2a/2c by LiPA assay were truly classified by NS5B phylogenetic analysis. ${ }^{40,41}$ In 2016, carrying out next generation sequencing (NGS) approach to detect RASs, we selected eight HCV GT1b positive patients. The HCV isolates were GT1b subtype by LiPA assay, except one classified ad mixed infection HCV GT1b/4. In contrast, analyzing majority rule NS3 NGS consensus and NS5B Sanger sequences, all samples were classified as HCV GT1b by phylogenetic analysis. Additionally, analyzing the same isolates we found naturally occurring RASs, such as V36L for boceprevir, telaprevir and simeprevir (1/8), I132V for telaprevir (4/8), V170I for grazoprevir, and $\mathrm{C} 316 \mathrm{~N}$ for dasabuvir $(2 / 8){ }^{42}$

Resistance testing for treatment of $\mathrm{HCV}$ is suggested for $\mathrm{HCV}$ GT1a at Q80K position on NS3 target region ${ }^{10}$ and HCV GT1a/ HCV3 at 28, 30,31, 58 and 93 positions on NS5A target region, ${ }^{10,14}$ in which the presence of RASs are clinically relevant for treatment choice and outcome during the first line therapy and retreatment. ${ }^{43}$ Indeed, patients whose HCV harbors RASs on NS5A are very difficult to re-treat, ${ }^{18}$ because these mutations persist for years after failure for genetic reasons not yet clarified. ${ }^{12}$ This kind of situations opens a new possibility of transmission of HCV resistant strains. As already well demonstrated for human immunodeficiency virus, spreading of transmitted drug resistance can compromise treatment response, highlighting the importance to study local epidemics. ${ }^{44}$ Obviously, emergent resistance to DAA in the setting of ongoing transmission is a significant obstacle against eradication of HCV infection. Last year, Cuypers et al. identified spread and transmission networks of HCV GT1a infection harboring Q80K by phylogenetic studies. ${ }^{45}$ These analyses suggested cost-effectiveness of resistance testing in patients eligible for simeprevir therapy. ${ }^{45}$

Finally, RASs should be carefully interpreted in clinical practice from a virological point of view. In the diagnostic routine, such test needs a better access and an easy interpretation. At present, geno2pheno (HCV) (g2p[HCV]) web-service supports the analysis of sequences of the target regions and can predict possible resistance against DAAs. This tool is easy to use, after the upload of sequence, the rules page allows to list RAS, substitutions at RAS position (RAPs) and fitness-associated substitutions related to drug response supported by scientific papers and clinical trials. ${ }^{46}$

\section{THIRD QUESTION: “CAN NGS APPROACH IM- PROVE DETECTION OF MINOR VIRAL POPU- LATION SO AS TO IMPROVE PREDICTION OF CLINICAL RESPONSE?"}

Currently, RASs detected at a lower frequency in minor populations are not believed to impact on SVR, because they usually reduce fitness compared with wild-type viruses. ${ }^{12,47}$ However, detection of RASs, RAPs and fitness-associated substitutions on the three eligible target regions are affected by unavailability of a standardized commercial test and analysis based on population sequencing with a cut-off of $15 \%$. It is currently unknown whether detection of mutations to below this cut-off may have an impact in clinical practice and many problems still exist.

First, in a limited number of laboratories in Europe and in other countries the test is hard-covered (in house assays), and a lack of a global consensus exists in the primers choice and reaction conditions to amply eligible target regions. ${ }^{10}$ Second, Sanger method is not enough to determine a tailored treatment due to high variability of virus. In fact, HCV is a viral quasispecies ${ }^{48}$ so the high replication rate $\left(10^{12}\right.$ virions per day) and an error-prone NS5B RNA-dependent RNA-polymerase contribute to generate mutations in $\mathrm{HCV}$ genome ${ }^{40}$ and closely related viral variants during infection. In the new era of DAAs, drug pressure could select for minor viral populations carrying RASs plus fitness-associated substitutions which can influence the outcome of treatment. ${ }^{12}$ Even if general data suggest that NGS sequencing (cut-off $\geq 1 \%$ ) does not improve resistance-based prediction compared with sequencing depths of $\geq 15 \%$, several studies are ongoing to compare deep sequencing and direct sequencing and to develop specific NGS analysis workflow. In 2016, we reported a combination of Ion Personal Genome Machine (PGM) Sequencer in conjunction with a freely available software to analyze nucleotide mutations, ranging from $0.05 \%$ to $10.9 \%$, in hot spot positions. ${ }^{42}$ In the same year, PGM approach $(<20 \%)$ was used to assess the frequencies of RAS at baseline in HCV1a and HCV1b in DAA-naive patients, showing deep sequencing as good method to detect RAS in the 
minor viral population in an easier and complete way. ${ }^{49}$

Thirty-five phase I, II, and III studies were analyzed by Zeuzem et al. to determine NS5A RASs effect on outcomes of treatment. ${ }^{50}$ A higher prevalence of RASs detection was observed at $1 \%$ sensitivity cut-offs, declining in sensitivity at 15\%. However, this last cut-off was the most discriminant. Paolucci et al., in 2017, reported a study where deep sequencing with cut-off values of $0.5 \%$ and $1 \%$ detected $66.7 \%$ of RASs at baseline, whereas direct sequencing detected only $33.3 \% .^{51}$ In recent clinical trials, such as POLARIS $-1^{27}$ and MAGELLAN, ${ }^{28}$ NGS approach with a sensitivity threshold $1 \%$ and $2 \%$, respectively, was used to detect RASs at baseline or at the time of virological failure to prove the impact of resistance during pan-genotypic treatment. In the POLARIS-1 study, five patients had selected RAS on NS3 or NS5A regions at failure using a sensitivity threshold of $1 \% .{ }^{27}$ The $2 \%$ of detection threshold identified V36M and M28G substitutions in NS3 and NS5A, respectively, in the MAGELLAN study. ${ }^{28}$

\section{CONCLUSION}

Even if the latest generation of IFN-free regimens are better tolerated, randomized clinical trials are not fully informative in the clinical practice because patients are selected based on strict criteria and number of patients is still limited with regards to several research questions under study. In particular, it is necessary to increase the real-world data to demonstrate the potential role of RASs and polymorphisms detected below cut-off $15 \%$ in treated or experienced patients. If RAS became dominant under drug pressure, HCV infected patients will benefit from minor viral population analysis to optimize treatment regimens with a tailored approach. Since eradication is not a mere dream today, fine tuning of treatment is necessary to make this objective even more realistic. $^{52}$

\section{Authors' contribution}

All authors have contributed to the design and the writing of the review.

\section{Conflicts of Interest}

No competing interests were disclosed.

\section{REFERENCES}

1. World Health Organization. Global hepatitis report 2017. World Health Organization. Geneva: World Health Organization, 2017:83.

2. Calvaruso V, Petta $S$, Craxì A. Is global elimination of HCV realistic? Liver Int 2018;38 Suppl 1:40-46.

3. Borgia SM, Hedskog C, Parhy B, Hyland RH, Stamm LM, Brainard $D M$, et al. Identification of a novel hepatitis $C$ virus genotype from Punjab, India - expanding classification of hepatitis C virus into 8 genotypes. J Infect Dis 2018;218:1722-1729.

4. Smith DB, Bukh J, Kuiken C, Muerhoff AS, Rice CM, Stapleton JT, et al. Expanded classification of hepatitis $C$ virus into 7 genotypes and 67 subtypes: updated criteria and genotype assignment web resource. Hepatology 2014;59:318-327.

5. Domingo E, Sheldon J, Perales C. Viral quasispecies evolution. Microbiol Mol Biol Rev 2012;76:159-216.

6. Kartashev V, Döring M, Nieto L, Coletta E, Kaiser R, Sierra S, et al. New findings in HCV genotype distribution in selected West European, Russian and Israeli regions. J Clin Virol 2016;81:82-89.

7. Liberto MC, Marascio N, Zicca E, Matera G. Epidemiological features and specificities of HCV infection: a hospital-based cohort study in a university medical center of Calabria region. BMC Infect Dis 2012;12 Suppl 2:S4

8. Marascio N, Matera G, Quirino A, Giancotti A, Barreca GS, Lamberti $A G$, et al. Eleven-year distribution pattern of hepatitis $C$ virus in southern Italy. J Pathog 2012;2012:631095.

9. Chhatwal J, Chen Q, Ayer T, Bethea ED, Kanwal F, Kowdley KV, et al. Hepatitis $C$ virus re-treatment in the era of direct-acting antivirals: projections in the USA. Aliment Pharmacol Ther 2018;47:1023-1031.

10. European Association for the Study of the Liver. EASL recommendations on treatment of hepatitis C 2016. J Hepatol 2017;66:153-194.

11. Bartlett SR, Grebely J, Eltahla AA, Reeves JD, Howe AYM, Miller V, et al. Sequencing of hepatitis $C$ virus for detection of resistance to direct-acting antiviral therapy: a systematic review. Hepatol Commun 2017;1:379-390.

12. Pawlotsky JM. Hepatitis C virus resistance to direct-acting antiviral drugs in interferon-free regimens. Gastroenterology 2016;151:7086

13. Korean Association for the Study of the Liver. KASL clinical practice guidelines: management of hepatitis C. Clin Mol Hepatol 2016;22:76-139.

14. American Association for the Study of Liver Diseases (AASLD). HCV Guidance: Recommendations for Testing, Managing, and Treating Hepatitis C. AASLD web site, <http://www.hcrguidelines.org>. Accessed 16 Mar 2018.

15. Geddawy A, Ibrahim YF, Elbahie NM, Ibrahim MA. Direct acting anti-hepatitis $C$ virus drugs: clinical pharmacology and future direction. J Transl Int Med 2017:5:8-17. 
16. Asselah T, Boyer N, Saadoun D, Martinot-Peignoux M, Marcellin P. Direct- acting antivirals for the treatment of hepatitis $C$ virus infection: optimizing current IFN-free treatment and future perspectives. Liver Int 2016;36 Suppl 1:47-57.

17. Omata M, Kanda T, Wei L, Yu ML, Chuang WL, Ibrahim A, et al. APASL consensus statements and recommendation on treatment of hepatitis C. Hepatol Int 2016;10:702-726.

18. Molino S, Martin MT. Hepatitis C virus resistance testing in genotype 1: the changing role in clinical utility. Ann Pharmacother 2017;51:811-816.

19. Bagaglio S, Uberti-Foppa C, Morsica G. Resistance mechanisms in hepatitis C virus: implications for direct-acting antiviral use. Drugs 2017;77:1043-1055

20. Ito J, Suda G, Yamamoto Y, Nagasaka A, Furuya K, Kumagai K, et al. Prevalence and characteristics of naturally occurring sofosbuvir resistance-associated variants in patients with hepatitis $C$ virus genotype 1b infection. Hepatol Res 2016;46:1294-1303.

21. Li Z, Zhang Y, Liu Y, Shao X, Luo Q, Cai Q, et al. Naturally occurring drug resistance associated variants to hepatitis $C$ virus direct-acting antiviral agents in treatment-naive HCV genotype $1 \mathrm{~b}$-infected patients in China. Medicine (Baltimore) 2017;96:e6830.

22. Cuypers L, Ceccherini-Silberstein F, Van Laethem K, Li G, Vandamme AM, Rockstroh JK. Impact of HCV genotype on treatment regimens and drug resistance: a snapshot in time. Rev Med Virol 2016;26:408-434

23. Cho BW, Kim SB, Song IH, Lee SH, Kim HS, Lee TH, et al. Efficacy and safety of daclatasvir plus asunaprevir for Korean patients with HCV genotype Ib infection: a retrospective multi-institutional study. Clin Mol Hepatol 2017;23:51-56.

24. Kliemann DA, Tovo CV, Gorini da Veiga AB, Machado AL, West J. Genetic barrier to direct acting antivirals in HCV sequences deposited in the European databank. PLoS One 2016;11:e0159924.

25. Ferenci P, Bernstein D, Lalezari J, Cohen D, Luo Y, Cooper C, et al. $A B T-450 / r-o m b i t a s v i r$ and dasabuvir with or without ribavirin for HCV. N Engl J Med 2014;370:1983-1992.

26. Marascio N, Pavia G, Romeo I, Talarico C, Di Salvo S, Reale M, et al. Real-life 3D therapy failure: analysis of NS5A 93H RAS plus 108K polymorphism in complex with ombitasvir by molecular modeling. J Med Virol 2018;90:1257-1263.

27. Struble K, Chan-Tack K, Qi K, Naeger LK, Birnkrant D. Benefit-risk assessment for sofosbuvir/velpatasvir/voxilaprevir based on patient population and hepatitis C virus genotype: U. S. Food and Drug Administration's evaluation. Hepatology. 2017 Oct 23. doi: 10.1002/ hep.29601.

28. Poordad F, Pol S, Asatryan A, Buti M, Shaw D, Hézode C, et al. Glecaprevir/Pibrentasvir in patients with hepatitis $C$ virus genotype 1 or 4 and past direct-acting antiviral treatment failure. Hepatology 2018;67:1253-1260.
29. Carrion AF, Martin P. Glecaprevir + pibrentasvir for treatment of hepatitis C. Expert Opin Pharmacother 2018;19:413-419.

30. Pham LV, Ramirez S, Gottwein JM, Fahnøe U, Li YP, Pedersen J, et al. HCV genotype 6a escape from and resistance to velpatasvir, pibrentasvir, and sofosbuvir in robust infectious cell culture models. Gastroenterology 2018;154:2194-2208.e12.

31. Younossi ZM, Stepanova M, Jacobson IM, Asselah T, Gane EJ, Lawitz $E$, et al. Sofosbuvir and velpatasvir with or without voxilaprevir in direct-acting antiviral-naïve chronic hepatitis C: patientreported outcomes from POLARIS 2 and 3. Aliment Pharmacol Ther 2018:47:259-267.

32. Polilli E, Cento V, Restelli U, Ceccherini-Silberstein F, Aragri M, Di Maio VC, et al. Consequences of inaccurate hepatitis $C$ virus genotyping on the costs of prescription of direct antiviral agents in an Italian district. Clinicoecon Outcomes Res 2016;8:467-473. eCollection 2016.

33. Strazzulla A, Costa C, Pisani V, De Maria V, Giancotti F, Di Salvo S, et al. Present, old and future strategies for anti-HCV treatment in patients infected by genotype-1: estimation of the drug costs in the Calabria Region in the era of the directly acting antivirals. BMC Infect Dis 2014;14 Suppl 5:S3.

34. Taherkhani R, Farshadpour F. Global elimination of hepatitis C virus infection: progresses and the remaining challenges. World J Hepatol 2017:9:1239-1252.

35. Torti C, Zazzi M, Abenavoli L, Trapasso F, Cesario F, Corigliano D, et al. Future research and collaboration: the "SINERGIE" project on HCV (South Italian Network for Rational Guidelines and International Epidemiology). BMC Infect Dis 2012;12 Suppl 2:S9.

36. Marascio N, Liberto M, Barreca G, Zicca E, Quirino A, Lamberti A, et al. Update on epidemiology of HCV in Italy: focus on the Calabria Region. BMC Infect Dis 2014;14 Suppl 5:S2.

37. Guadagnino V, Stroffolini T, Caroleo B, Menniti Ippolito F, Rapicetta $M$, Ciccaglione $A R$, et al. Hepatitis $C$ virus infection in an endemic area of Southern Italy 14 years later: evidence for a vanishing infection. Dig Liver Dis 2013;45:403-407.

38. Marascio N, Torti C, Liberto M, Focà A. Update on different aspects of HCV variability: focus on NS5B polymerase. BMC Infect Dis 2014;14 Suppl 5:S1.

39. Stelzl E, Appel HM, Mehta R, Marins EG, Berg J, Paar C, et al. Evaluation of the new cobas(R) HCV genotyping test based on real-time PCRs of three different HCV genome regions. Clin Chem Lab Med 2017;55:517-521.

40. Ciccozzi M, Equestre M, Costantino A, Marascio N, Quirino A, Lo Presti $A$, et al. Hepatitis $C$ virus genotype $4 d$ in Southern Italy: reconstruction of its origin and spread by a phylodynamic analysis. J Med Virol 2012;84:1613-1619.

41. Marascio N, Ciccozzi M, Equestre M, Lo Presti A, Costantino A, Cella $E$, et al. Back to the origin of HCV 2c subtype and spreading to the 
Calabria region (Southern Italy) over the last two centuries: a phylogenetic study. Infect Genet Evol 2014;26:352-358.

42. Marascio N, Pavia G, Strazzulla A, Dierckx T, Cuypers L, Vrancken B, et al. Detection of natural resistance-associated substitutions by ion semiconductor technology in HCV1b positive, direct-acting antiviral agents-naïve patients. Int J Mol Sci 2016;17. pii: E1416.

43. Sarrazin C, Dvory-Sobol H, Svarovskaia ES, Doehle BP, Pang PS, Chuang $S M$, et al. Prevalence of resistance-associated substitutions in HCV NS5A, NS5B, or NS3 and outcomes of treatment with ledipasvir and sofosbuvir. Gastroenterology 2016;151:501-512.e1.

44. Pineda-Peña AC, Schrooten Y, Vinken L, Ferreira F, Li G, Trovão NS, et al. Trends and predictors of transmitted drug resistance (TDR) and clusters with TDR in a local Belgian HIV-1 epidemic. PLoS One 2014;9:e101738.

45. Cuypers L, Vrancken B, Fabeni L, Marascio N, Cento V, Di Maio VC, et al. Implications of hepatitis C virus subtype 1a migration patterns for virus genetic sequencing policies in Italy. BMC Evol Biol 2017;17:70.

46. Kalaghatgi P, Sikorski AM, Knops E, Rupp D, Sierra S, Heger E, et al. Geno2pheno[HCV] - a web-based interpretation system to support hepatitis $C$ treatment decisions in the era of direct-acting antiviral agents. PLoS One 2016;11:e0155869.

47. Sarrazin C. The importance of resistance to direct antiviral drugs in HCV infection in clinical practice. J Hepatol 2016;64:486-504.

48. Martell M, Esteban JI, Quer J, Genescà J, Weiner A, Esteban R, et al. Hepatitis $C$ virus (HCV) circulates as a population of different but closely related genomes: quasispecies nature of HCV genome distribution. J Virol 1992;66:3225-3229.

49. Gaspareto KV, Ribeiro RM, de Mello Malta F, Gomes-Gouvêa MS, Muto NH, Romano CM, et al. Resistance-associated variants in HCV subtypes $1 \mathrm{a}$ and $1 \mathrm{~b}$ detected by lon Torrent sequencing platform. Antivir Ther 2016;21:653-660.

50. Zeuzem S, Mizokami M, Pianko S, Mangia A, Han KH, Martin R, et al. NS5A resistance-associated substitutions in patients with genotype 1 hepatitis $C$ virus: prevalence and effect on treatment outcome. J Hepatol 2017;66:910-918.

51. Paolucci S, Premoli M, Novati S, Gulminetti R, Maserati R, Barbarini $\mathrm{G}$, et al. Baseline and breakthrough resistance mutations in HCV patients failing DAAs. Sci Rep 2017;7:16017.

52. Torti C, Focà A, Carosi G. Towards HCV extinction with modern HCV treatment? "Yes we can!". BMC Infect Dis 2012;12 Suppl 2:S1. 